QoL was measured in 1,268 patients (45\% female) at 180 days after treatment randomization, using the Short Form-36 health survey. The mean age of female patients was significantly greater than that of male patients (73.4 years vs 68.4 years; $P<0.0001$ ). In addition, female patients were more likely to have atrial fibrillation, to have a lower premorbid functional status (as measured by the modified Rankin Scale [mRS]), and to be in a nursing home, than were male patients.

Overall, QoL scores for female patients were markedly lower than those for male patients; female patients scored particularly poorly on the domains relating to physical functioning, vitality and mental health. Even following adjustment for age, baseline systolic blood pressure, Scandinavian Stroke Scale score, history of myocardial infarction, stroke type, premorbid $\mathrm{mRS}$ score, premorbid residency (home/nursing home), and treatment group, female patients had considerably lower scores for physical functioning and mental health than did male patients. Notably, lower scores on the physical functioning and mental health QoL domains were associated with poor functional outcome at 6 months, as assessed by the $\mathrm{mRS}$ ( $P<0.0001$ for both).

Original article Gray LJ et al. (2007) Sex differences in quality of life in stroke survivors: data from the Tinzaparin in Acute Ischaemic Stroke Trial (TAIST). Stroke 38: 2960-2964

\section{Blood plasma biomarkers could predict Alzheimer's disease}

Early and definitive diagnosis of Alzheimer's disease (AD) could lead to better and moretargeted treatment for patients. Ray et al. have recently identified a group of plasma signaling proteins that reflect changes in the brain during the early stages of AD pathology. Levels of the 18 proteins in blood plasma can be used to identify patients with $A D$, or to predict the onset of $A D$ in presymptomatic patients, with close to 90\% accuracy.

First, the authors measured the relative abundance of 120 signaling proteins in an initial set of samples from 43 patients with $A D$ and 40 controls without dementia, and through statistical analysis found significantly different expression patterns of 19 proteins ( $q$-value $<3.4 \%$ ), from which a set of 18 predictors of AD was identified. When the predictors were then tested in independent samples from patients with $A D$ $(n=42)$ and other types of dementia $(n=11)$ and controls without dementia $(n=39)$, patients were classified with $89 \%$ accuracy $(P<0.001)$. Furthermore, in 47 patients with mild cognitive impairment, progression to $A D$ was predicted with $81 \%$ accuracy $(P<0.001)$ up to 6 years before clinical diagnosis. Functional profiling of the 18 predictor proteins pointed to involvement in systemic dysregulation of intracellular pathways of immune response, hematopoeisis and apoptosis.

Further tests are needed before this approach can be used in patients, but the identification of blood plasma biomarkers early in the course of $A D$ could open the door to more-effective interventions.

Original article Ray S et al. (2007) Classification and prediction of clinical Alzheimer's diagnosis based on plasma signaling proteins. Nat Med 13: 1359-1362

\section{Early exposure to mercury- containing vaccines has no consistent effect on neuropsychology}

Concerns have been expressed about the potentially negative neuropsychological effects of the mercury-containing preservative thiomersal (thimerosal) in pediatric vaccines; however, a recent cohort study found no evidence to indicate a causal relationship between deficits in neuropsychological functioning at 7-10 years and early exposure to thiomersal-containing vaccines.

The study used standardized tests to assess 42 neuropsychological outcomes in 1,047 children aged 7-10 years; autism-spectrum disorders were not assessed. Each child's exposure to mercury from thiomersal was determined from computerized immunization records, medical records, parent immunization records and parent interviews. The median cumulative exposure to mercury from thiomersal from birth to 7 months was $112.5 \mu \mathrm{g}$ (range $0-187.5 \mu \mathrm{g}$ ); 16 children had no thiomersal exposure during this period. After adjustment for potential confounding factors, few significant associations were found between test performance and exposure to thiomersal. Those associations that were detected were small, almost equally divided 